\title{
On-chip broadband spectral filtering using planar double high-contrast grating reflectors
}

\author{
Yu Horie, Amir Arbabi, and Andrei Faraon \\ T. J. Watson Laboratory of Applied Physics, California Institute of Technology, \\ 1200 E. California Blvd., Pasadena, CA 91125, USA
}

\begin{abstract}
We propose a broadband free-space on-chip spectrometer based on an array of integrated narrowband filters consisting of Fabry-Perot resonators formed by two high-contrast grating (HCG) based reflectors separated by a low-index thin layer with a fixed cavity thickness. Using numerical simulations, broadband tunability of resonance wavelengths was achieved only by changing the in-plane grating parameters such as period or duty cycle of HCGs while the substrate geometry was kept fixed. Experimentally, the HCG reflectors were fabricated on silicon on insulator (SOI) substrates and high reflectivity was measured, fabrication process for the proposed double HCG-based narrowband filter array was developed. The filtering function that can be spanned over a wide range of wavelengths was measured.
\end{abstract}

Keywords: Spectral filtering, high-contrast grating, on-chip spectrometer, narrowband optical filter, metasurface, high index contrast, sub-wavelength grating

\section{INTRODUCTION}

A spectrometer is one of the most important instruments in the field of optics. Integrated miniaturized spectrometers have been the subject of considerable recent interest due to their potential advantages including low-cost fabrication, device compactness, and high sensitivity. ${ }^{1}$ Motivated by this demand, several approaches have been investigated including tunable ring resonator-based optical circuits, ${ }^{2}$ photonic crystal cavity arrays ${ }^{3}$ and deterministic light scattering in a random media. ${ }^{4}$ For free-space spectroscopy, one of the attractive designs consists of an array of on-chip narrowband band-pass optical filters placed on a photodetector array. ${ }^{5}$ Collimated incident light beam with a broadband spectrum of interest is filtered out by the narrowband band-pass filters with different central wavelengths, and the corresponding photodetectors underneath is used to detect the power intensity of the light in the filtered spectrum, allowing to analyze the spectral information for the incident light. Such a narrowband optical filter may be implemented by using Fabry-Perot (FP) resonators formed by two highly reflective distributed Bragg reflectors (DBRs) with spatially varied cavity length so that each of the resonators has a different resonance wavelength. Gray-scale lithographic technology enables realization of the spatially varying DBR cavity thickness in a controllable way as reported in. ${ }^{6}$ However, such a high-end and expensive lithography technique is not readily available and will prevents the realization of the narrowband optical filter array in mass production.

In this contribution, we propose an array of narrowband filters that covers a wide range of wavelengths, based on an array of FP resonators formed by two-layers of high-contrast grating (HCG) reflectors Fig. 1(a). HCGs not only provide broadband reflection spectra comparable to DBRs thus being regarded as the thin-layer alternatives of DBRs, but also, by changing the grating geometry, it allows for engineering the reflection phase while the reflectivity is kept very high. ${ }^{7,8}$ As schematically shown in Fig. 1, each of the narrowband optical filters is made of two identical HCGs separated by a distance on the order of a wavelength. Only the portion of the incident light that is critically coupled to the resonator can be transmitted with $100 \%$ efficiency in theory. It is advantageous to replace DBRs with HCG reflectors because the resonance wavelengths of the filters can be tuned by modifying only the in-plane grating parameters such as the period or the duty cycle, while the vertical dimensions including the grating heights and the cavity length can be kept constant. The lateral dimensions of the grating, which determine the resonance wavelengths of the FP resonators, can be precisely controlled by the conventional lithography.

Further author information: (Send correspondence to A.F.)

A.F.: E-mail: faraon@caltech.edu, Telephone: 16263953086

High Contrast Metastructures IV, edited by Connie J. Chang-Hasnain, David Fattal,

Fumio Koyama, Weimin Zhou, Proc. of SPIE Vol. 9372, 937200 - C 2015 SPIE

CCC code: $0277-786 \mathrm{X} / 15 / \$ 18 \cdot$ doi: $10.1117 / 12.2080451$

Proc. of SPIE Vol. $9372937200-1$ 
(a)

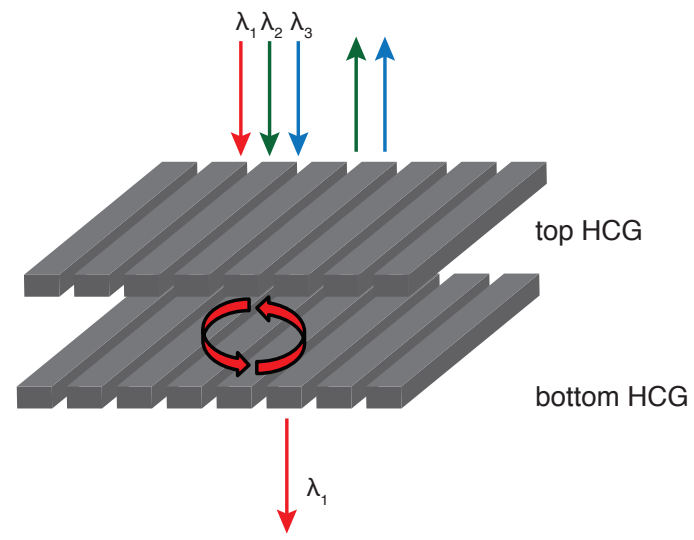

(b)

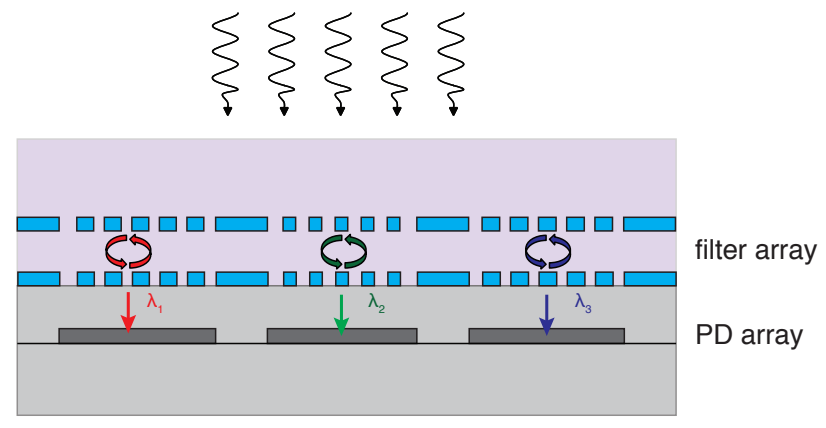

Figure 1. (a) Schematic illustration of double HCG reflectors which form a FP resonator. Each of the filter pixels is made of a similar planar double grating structure. (b) Schematic of an array of optical filters and an underlying photodetectors. Collimated broadband input light is filtered out by the narrowband filter array, and the intensity of the filtered light is detected by the corresponding photodetector pixel.

\section{METHOD AND RESULTS}

\subsection{Design of double HCG reflectors}

To investigate the operation of the double HCG-based filters, we begin with a broadband reflector design that operates at the telecommunication band. A HCG refers to a sub-wavelength periodic structure made of a highrefractive index material surrounded by a low-index matrix. Due to the sub-wavelength nature of the grating, there is no higher order diffraction for a normally incident light, and high-index contrast system makes the guided modes in the grating very dispersive, opening up unique properties such as broadband unity reflection or transmission for the grating. The details of operating principle for the HCG can be found in. ${ }^{9}{ }^{10}$ The HCG-based reflectors considered in this paper are $1 \mathrm{D}$ gratings made of $\mathrm{Si}$ and embedded in low-index materials such as $\mathrm{SiO}_{2}$ or SU-8, but the designs can be readily extended to other high contrast system or 2D periodic systems. We used the rigorous coupled-wave analysis (RCWA) technique ${ }^{11}$ to optimize the reflector design. The basic reflector designed for the normally incident TE-polarized light has a period of $880 \mathrm{~nm}$, duty cycle of 0.5 , and the height of $310 \mathrm{~nm}$. When two of such reflectors are placed in parallel and are separated by a $1.2 \mu \mathrm{m}$-thick SU-8 spacer layer, which satisfies the FP resonance condition, a single FP resonance within the highly reflective stopband is observed. The spacer layer thickness between the double HCG layers must be larger than half of the wavelength so that near-field coupling between the two HCG layers is avoided. Using RCWA technique, we calculated the resonance wavelength of the double HCG resonator as we varied the in-plane grating parameters of period and duty cycle, while keeping the vertical dimensions fixed. To ensure proper spectral filtering function, it is important to avoid the overlap of the FP resonance with other undesired resonances or the stopband edge of the HCG reflectors. Considering this issue, we found a set of optimized parameters, which provides a broadband operation for our spectral filtering design. The reflection spectra of the typical four different double HCG filters having different central wavelengths are plotted in Fig. 2. We found that the filtering operation over the wide range of $\Delta \lambda=70 \mathrm{~nm}(\Delta \lambda / \lambda=5 \%)$ with moderately high quality factors $\sim 10^{3}$ can be achieved in the $\mathrm{SU}-8 / \mathrm{Si} / \mathrm{SiO}_{2}$ configuration by only changing the lateral grating dimensions. Since the broader reflection bandwidth of HCG reflectors is given by higher index contrast, further improvement for the wavelengths covered can be designed using higher index contrast system. For instance, using a $\mathrm{SiO}_{2} / \mathrm{Si} / \mathrm{SiO}_{2}$ configuration designed for the normally incident TM-polarized light, we were able to obtain more broadband operation with $\Delta \lambda=120 \mathrm{~nm}(\Delta \lambda / \lambda=8 \%)$. It should be also noted that since the structure size scales with the wavelength, the proposed design should be applicable to any wavelengths of interest when high contrast system is available. 


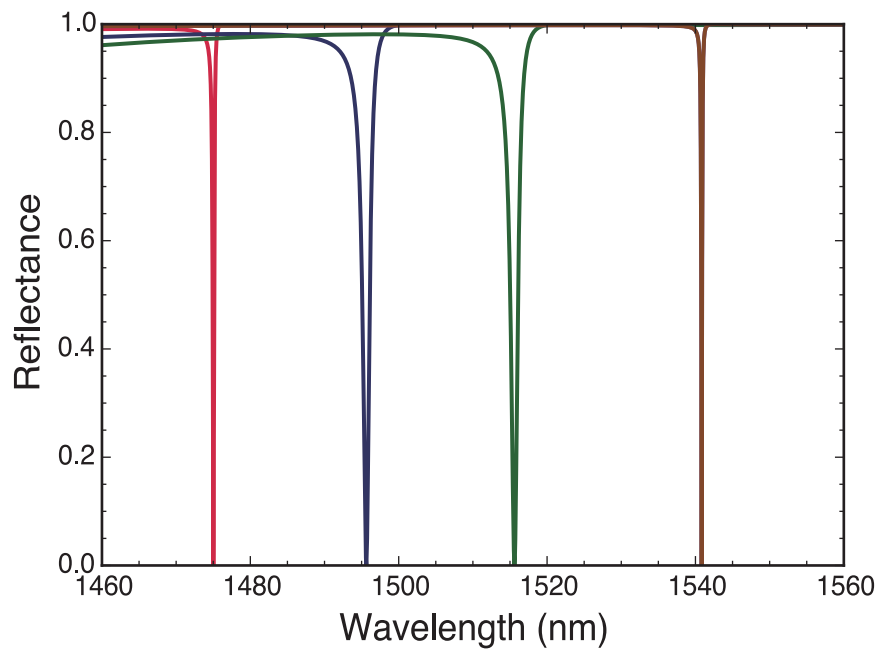

Figure 2. The calculated reflection spectra for a series of four different double HCG filter designs. The broadband operation can be achieved by only changing the in-plane grating geometry.

\subsection{Fabrication}

The double HCG-based filter arrays are fabricated on an SOI substrate with a Si device layer and $3 \mu \mathrm{m}$ buried oxide layer. First, the Si layer was thinned down to $310 \mathrm{~nm}$ using a thermal oxidation process followed by buffered oxide etch for the top oxidized Si. The grating pattern was defined by electron beam lithography using a positive resist (ZEP-520A) followed by inductively coupled plasma reactive ion etching (ICP-RIE) process using $\mathrm{SF}_{6} / \mathrm{C}_{4} \mathrm{~F}_{8}$ chemistry for patterning the first Si layer. The patterned Si was spin-coated by SU-8 2002 for planarization purpose for the subsequent amorphous $\mathrm{Si}(\mathrm{a}-\mathrm{Si}$ ) layer deposition. An a-Si layer was deposited with a thickness of $310 \mathrm{~nm}$ by plasma-enhanced chemical vapor deposition (PECVD) method. Another grating pattern was then defined for the second a-Si layer using the same lithography and etching processes but with an electron beam alignment along the initial mask. Finally, the whole structures were cladded with SU-8 again for the planarization purpose. The entire fabrication process flow is schematically shown in Fig. 3(a). The typical cross-sectional image for the fabricated double HCG layers is shown in Fig. 3(b).

\subsection{Experimental results}

We characterized the fabricated filter array by measuring their reflectivity spectra. The reflection spectra were measured by scanning the wavelengths of our tunable laser and collecting the reflected light from the devices using an InGaAs photodetector. Using an objective lens, the collimated and linearly-polarized beam from the laser is focused onto each filter from the normal direction, where the beam width at focus is expected to be about $60 \mu \mathrm{m}$. The measured reflection spectra from the double HCG filters with three different designs where varying the duty cycles of the Si gratings are shown in Fig. 4. The filtering function due to the FP resonance between the double HCGs was observed and the resonance wavelengths in the measured spectra and the simulated spectra showed a good agreement. The measured quality factors for the FP resonators ranged around the order of $10^{3}$. We also measured the transmission spectra in order to confirm that the beam passing through the filters is transmitted to the backside of the SOI substrates. Although it is not plotted in the manuscript, the transmission peaks, which correspond to the peaks found in the reflection spectra, were observed. However, the transmitted absolute power was low due to the reflection at the backside as well as the unpolished surface on the backside of the SOI substrates. 
(a)

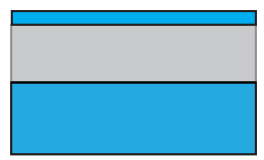

SOI

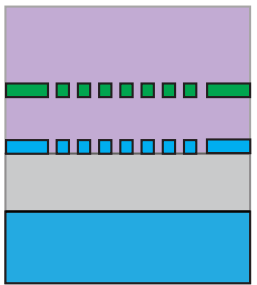

cladding with SU-8

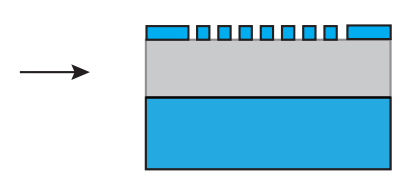

pattern 1st Si layer

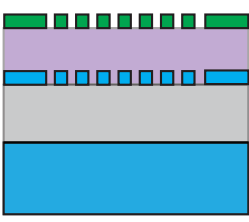

pattern 2nd a-Si layer

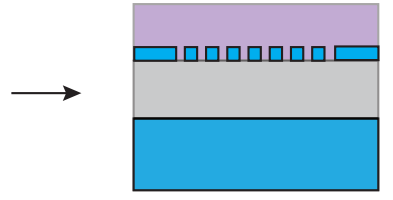

planarization with SU-8

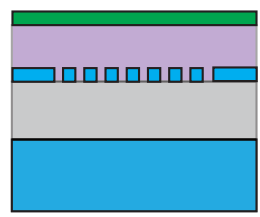

a-Si deposition

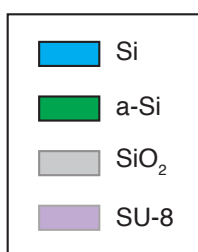

(b)

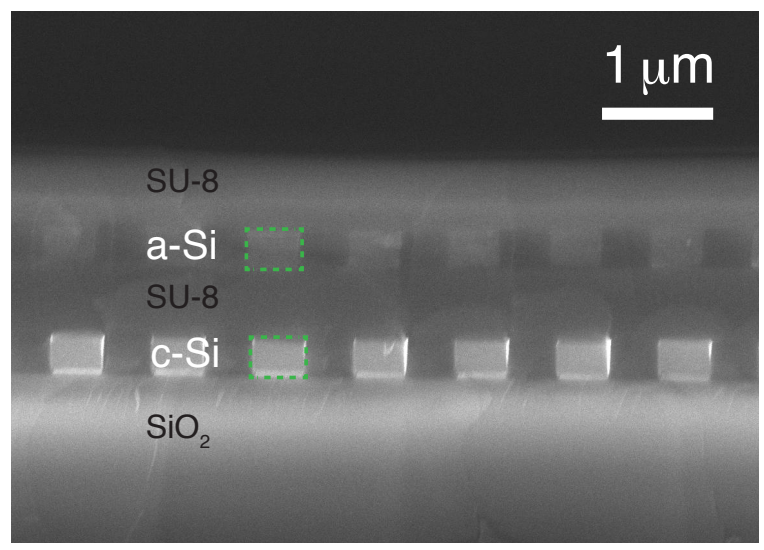

Figure 3. (a) Fabrication process for double HCG narrowband filter arrays on a SOI substrate. The central wavelength for each double HCG-based filter can be tuned by precisely controlling the in-plane dimensions of the HCG layers. Unlike conventional DBR-based configurations, it is not required to control the vertical dimensions to tune the resonance wavelengths of narrowband filters. (b) The typical cross-sectional SEM image of fabricated double HCG-based filters. 


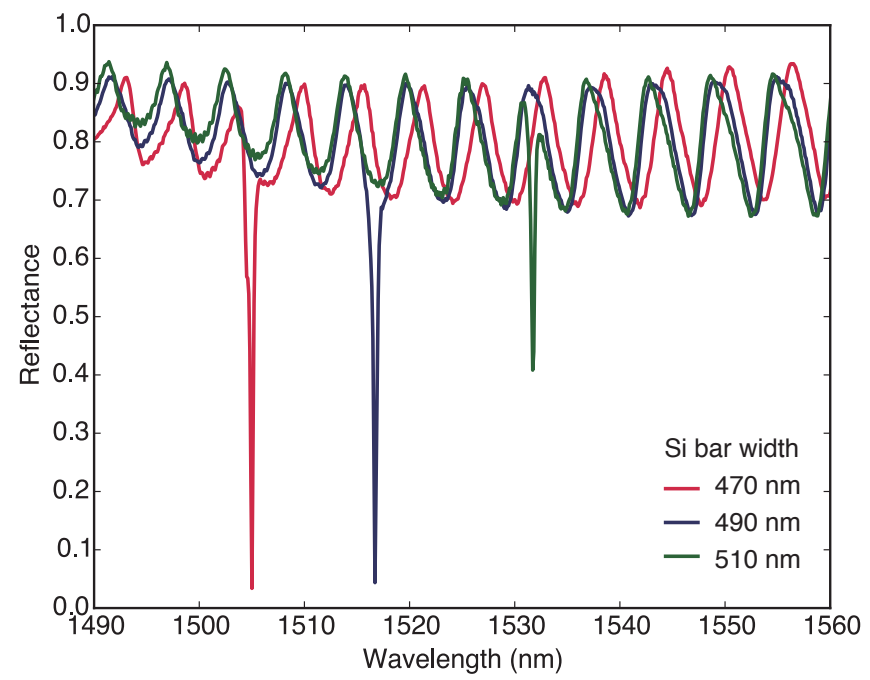

Figure 4. The measured reflection spectra for the FP filters with three different designs. By changing the duty cycle of the HCG layers (determines the high-index Si bar widths, the wavelengths of FP resonances can be tuned. The background spectrum oscillation comes from the measurement setup, not from the filters. By precisely controlling the duty cycle of the HCGs, filter arrays with finer resolution will be realized.

\section{CONCLUSION}

In conclusion, we propose and experimentally demonstrate a spectral filtering based on a narrowband band-pass filter array, using double HCG reflectors. Each filter is narrow-band but the center wavelength can span a wide range of spectrum just by varying the in-plane parameters of the HCG reflector, which will be precisely controllable by conventional lithography. By integrating such planar narrowband filter array onto underlying photodetector array, compact on-chip spectrometers with low cost can be realized.

\section{REFERENCES}

1. Momeni, B., Yegnanarayanan, S., Soltani, M., Eftekhar, A. A., Shah Hosseini, E., and Adibi, A., "Silicon nanophotonic devices for integrated sensing," J. Nanophotonics 3(1), 031001 (2009).

2. Kyotoku, B., Chen, L., and Lipson, M., "Sub-nm resolution cavity enhanced microspectrometer," Opt. Express 18(1), 102-107 (2010).

3. Gan, X., Pervez, N., Kymissis, I., Hatami, F., and Englund, D., "A high-resolution spectrometer based on a compact planar two dimensional photonic crystal cavity array," Appl. Phys. Lett. 100(23), 231104 (2012).

4. Redding, B., Liew, S. F., Sarma, R., and Cao, H., "Compact spectrometer based on a disordered photonic chip," Nature Photon. 7, 746-751 (2013).

5. Wang, S.-W., Xia, C., Chen, X., Lu, W., Li, M., Wang, H., Zheng, W., and Zhang, T., "Concept of a high-resolution miniature spectrometer using an integrated filter array," Opt. Lett. 32(6), 632-634 (2007).

6. Xiao, J., Song, F., Han, K., and Seo, S. W., "Fabrication of CMOS-compatible optical filter arrays using gray-scale lithography," J. Micromech. Microeng. 22, 025006 (2012).

7. Chang-Hasnain, C. J. and Yang, W., "High-contrast gratings for integrated optoelectronics," Adv. Opt. Photonics 4(3), 379-440 (2012).

8. Fattal, D., Li, J., Peng, Z., Fiorentino, M., and Beausoleil, R. G., "Flat dielectric grating reflectors with focusing abilities," Nature Photon. 4(7), 466-470 (2010).

9. Karagodsky, V., Sedgwick, F. G., and Chang-Hasnain, C. J., "Theoretical analysis of subwavelength high contrast grating reflectors," Opt. Express 18(16), 16973-16988 (2010). 
10. Karagodsky, V. and Chang-Hasnain, C. J., "Physics of near-wavelength high contrast gratings," Opt. Express 20(10), 10888-10895 (2012).

11. Liu, V. and Fan, S., "S4: A free electromagnetic solver for layered periodic structures," Comput. Phys. Commun. 183(10), 2233-2244 (2012). 